\title{
IMPROVED VIBRATION CONTROL OF A SMART BEAM BY ENERGY TRANSFER
}

\author{
Aïda Chérif ${ }^{1}$, Hadjira Attoui ${ }^{2}$, Djamila Zehar $^{3}$, Khalissa Behih $^{4}$
}

\begin{abstract}
The modal synchronized switch damping on inductor approach SSDI-Max, maximize the self-generated voltage amplitude, by a proper definition of the switch instants, according to the chosen targeted mode. This paper presents a performance analysis of a new global approach, for improved vibration damping of smart structure based on global energy redistribution by means of a piezoelectric element network, this network topology, using diodes called SSDD-Max "Synchronized Switch Damping with Diode-Max". In this paper, simulations are performed on a model representative of a smart beam. The damping results are given in the case of multimodal, pulse excitations.
\end{abstract}

Keywords - Piezoelectric Materials, Vibration Control, Energy Transfer, Smart Beam, SSDI-Max, SSDD-Max

\section{INTRODUCTION}

The material fatigue, shortening the operation life and also increasing the maintenance cost, is a result of the mechanical vibrations of structure. Consequently, vibration control is an important issue in the mechanical engineering. In various industrial domains such as manufacturing, infrastructure engineering, instrumentation, automotive, aerospace, electronic communications, computer, and other high-technical domains, significant advances have been made in structural control researches, to avoid the undesirable vibration problems.

In the past decades, many approaches using piezoelectric materials have been developed for vibration suppression in smart structures. One can underline the approaches, using piezoelectric shunt, which consist in adding simple electronic circuits comprising inductors, capacitors, and resistors. They are simple to design and work without any additional power amplifiers.

More recently, semi-active vibration control methods have received much attention for structural control. Compared to the active vibration control methods, it only requires a small external energy for operation of the vibration control, besides this advantage, it also would not lead to modal spillover. The Synchronized Switch Damping (SSD) techniques have been developed by Richard and Guyomar [1][2]. SSD techniques reach a good trade-off between the simplicity, external energy requirement and vibration control performance. This non-linear technique consists of connecting intermittently the piezoelectric element to a passive shunt (small resistance or inductor) each time the voltage (or strain) reaches an extremum.

Based on a modal model and SSD techniques of the controlled structure, the so called Modal-SSDI has been developed [3][4]. This technique consists of synchronizing the switch sequence on a given modal coordinate instead of the voltage. It therefore combines the simplicity, the robustness and low power operation of SSDI techniques, with the possibility of mode targeting and precision of active control strategies. The SSDI-Max method [5][6][7] derived from Modal-SSDI was developed to improve the damping performance by the maximization of the selfgenerated voltage amplitude using an enhanced switching sequence relying on both the desired modal coordinate and the voltage signals.

Wu et al [8] developed a global approach based on global energy redistribution by means of a piezoelectric element network, so called SSDD (Synchronized Switch Damping with Diode) network apply on a plate model. In this paper

${ }^{1}$ LAS Laboratory, Faculty of technology, University of Setif 1, 19000 Setif, Algeria

${ }^{2}$ QUERE Laboratory, Faculty of technology, University of Setif 1, 19000 Setif, Algeria

${ }^{3}$ Electromechanic department, University of BBA, Algeria.

${ }^{4}$ LSI Laboratory, Faculty of technology, University of Setif 1, 19000 Setif, Algeria 
we propose an improved technical SSDD based on modal technical SSDI-Max, this new technique is called SSDDMax. The presented simulations are performed on a model representative of a smart beam. The damping results are given in the case of multimodal, pulse excitations.

\section{SMART STRUCTURE MODELING}

The electromechanical principle of a vibrating structure can be described as a single degree of freedom (DOF) oscillator. Indeed, if the mechanical structure vibrates with a linear behavior on a single mode, the electromechanical system can be represented by an equivalent model: mass+piezo+spring+damper, as shown in Fig.1.

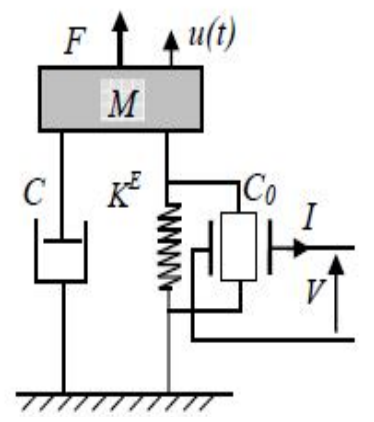

Figure 1. Schematic representation of the electromechanical model [9]

The electromechanical behavior equations of a smart structure using usual assumption are as follows: $m \ddot{u}+c \vec{u}+k^{\bar{E}} u=-\alpha V+\beta F$

$I=\alpha \dot{u}-C_{0} b$

With $u$ the nodal displacement vector, $m, c$, and $k^{E}$ are the mass, damping, and stiffness matrices, respectively, when the piezoelectric patches are in short circuit, $\alpha$ is the electromechanical coupling matrix, $V$ is voltage vector of the i piezoelectric patches, $I$ is the electric current vector, and $C_{0}$ is the diagonal capacitance matrix. $F$ is the force applied to the system.

Using the following variable change where $\phi$ is the mode shape matrix limited to $n$ modes and $q$ the modal displacement vector:

$\delta=\phi q$

The equations (1) and (2) can be well represented, by the projection in the modal basis by:

$M \ddot{q}+C \dot{q}+K^{E} q=-\theta V+\beta F$

$I=\theta^{t} \dot{q}-C_{0} \dot{V}$

Where $\theta$ is the modal electromechanical coupling matrix with a [n,i] matrix size. $\theta$ is defined as follows

$\theta=\phi^{t} . \alpha$

Where $M, C$, and $K^{E}$ are the mass, damping, and stiffness modal matrices, respectively. The structure is assumed to be lightly damped, with proportional damping and decoupled modes. Equation (4) is normalized in order to have the modal mass matrix equal to identity. The modal matrices can therefore be written as a function of $\xi$ the modal damping vector, $\omega^{\bar{E}}$ the short-circuit frequency vector, and $\omega^{B}$ the open circuit frequency vector as follows

$M=I_{d} ; C=2 \operatorname{diag}(\xi) \operatorname{diag}\left(\omega^{D}\right) ; K^{E}=\operatorname{diag}\left(\left(\omega^{E}\right)^{2}\right)$

By separating the actuators and sensor voltages, namely, $V_{\mathrm{a}}$ and $V_{\mathrm{s}}$, respectively, equation (4) becomes

$M \ddot{q}+C \dot{q}+K^{E} q=-\theta_{a} V_{a}-\theta_{s} V_{s}+\beta F$

In an open circuit or when the sensor voltage is monitored with a voltage amplifier, sensor intensity is null, therefore

$\theta_{s}^{t} q-C_{0 s} V_{s}=0$

And by reintroducing equation (9) in equation (8)

$M \ddot{q}+C \dot{q}+\left(K^{E}+\theta_{s}\left(C_{0 s}\right)^{-1} \theta_{s}^{t}\right) q=-\theta_{a} V_{a}+\beta F$

Linear systems (10) and (9) can be written in modal state under the form 
$\left\{\begin{array}{c}\dot{x}=A x+B u \\ y=C x\end{array} \quad x=\left[\begin{array}{c}q \\ \dot{q}\end{array}\right]\right.$

With $x$ being the state vector, $u=\left[F, V_{a}\right]$ is the control vector, $y=\left[q, \dot{q}, V_{s}\right]$ is the output vector, and $A, B$, and $C$ are the state matrices

$$
A=\left[\begin{array}{cc}
0 & I_{d} \\
-M^{-1}\left(K^{E}+\theta_{s} C_{0 s}^{-1} \theta_{s}^{t}\right) & -M^{-1} C
\end{array}\right], B=\left[\begin{array}{cc}
0 \\
+M^{-1} \beta & -M^{-1} \theta_{a}
\end{array}\right], C=\left(\begin{array}{cc}
I_{d} & 0 \\
0 & I_{d} \\
C_{0 s}^{-1} \theta_{s}^{t} & 0
\end{array}\right)
$$

$V_{a}$ is calculated by the following relation:

$$
V_{a}=C_{0 a}^{-1} \theta_{a}^{t} q
$$

$C_{0 a}$ is the capacity of actuator, and the matrix $C_{0 s}$ is capacity sensors.

Integrating the variable over the time and multiplying both terms of (1) by the velocity $\boldsymbol{u}$, the energy equation could be written as (14):

$$
\int m u \dot{u} d t+\int \operatorname{cu}^{2} d t+\int k{ }^{E} u \dot{u} d t+\int \alpha V \dot{u} d t=\int F u d t
$$

The provided energy is distributed into kinetic energy, potential energy, mechanical energy losses and transferred energy.

The integration over a finite number of periods in the steady state leads to (15)

$$
\left[\frac{1}{2} m \dot{u}^{2}\right]_{0}^{T}+\left[\frac{1}{2} K^{E} u^{2}\right]_{0}^{T}+\int_{0}^{T} c \dot{u}^{2} d t+\int_{0}^{T} \alpha V \dot{u} d t=\int_{0}^{T} F \dot{u} d t
$$

If we consider as initial conditions that the displacement $u$ and velocity are zero. (15) leads to (16). The structure energy terms are defined in the equation (16)

$\frac{1}{2} m u^{2}+\frac{1}{2} k_{E} u^{2}+\int_{0}^{T} c u^{2} d t+\int_{0}^{T} \alpha V u d t=\int_{0}^{T} F u d t$

Table-1 Structure Energy terms [10]

\begin{tabular}{|l|l|}
\hline Definition & Energy \\
\hline$E_{f}=\int_{0}^{T} F \dot{u} d t$ & Supplied Energy \\
\hline$E_{R}=\frac{1}{2} m \dot{u}^{2}$ & Kinetic Energy \\
\hline$E_{p}=\frac{1}{2} k_{E} u^{2}$ & Potential elastic Energy \\
\hline$E_{V}=\int_{0}^{T} a u^{2} d t$ & Viscous damping Energy \\
\hline$E_{S}=-\int_{0}^{T} \alpha V u \dot{u} d t$ & Transferred Energy \\
\hline
\end{tabular}

The transferred energy $\int \alpha V \dot{u} d t$ corresponds to the part of the mechanical energy, which is converted into electrical one. The transferred energy is also called converted energy or extracted energy. This energy is the sum of the electrostatic energy stored in an electrical element (piezoelectric capacitor $C_{0}$ ) and the energy dissipated in the electric circuit connected to the piezoelectric element (dissipated in the circuit by joule effect).

\section{SMART-STRUCTURE DEFINITION}

The structure that will be used, in the following simulations and analyses is a clamped-free smart beam, composed of a beam in dur aluminium and four P188 piezoelectric patches bonded on the beam. One is used as actuator, two others as sensors, and the last for the excitation. The characteristics of the smart structure are given in table 2. Fig.2 illustrates this beam. This structure has been identified according to the previously described model. The measurement process and parameter identification is described in [11]. Table 3 summarizes the frequencies of the three modes considered in the model. 


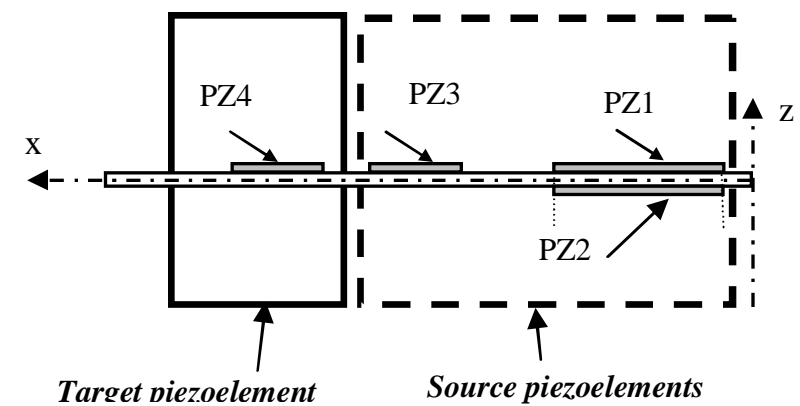

Figure 2. Schematic of the smart-beam implemented and used in this study [11]

Table-2 Materials properties [11]

\begin{tabular}{|c|c|c|}
\hline \multicolumn{3}{|l|}{ Aluminium beam } \\
\hline density $: \rho=2800 \mathrm{Kg} \cdot \mathrm{m}^{-3}$ & Young modulus $\mathrm{E}=7 \times 10^{11} \mathrm{~Pa}$ & Poisson ratio $v=0.3$ \\
\hline \multicolumn{3}{|c|}{ Piezoelectric ceramic : P188 Ceramic } \\
\hline \multicolumn{3}{|c|}{ density $\rho c=7700 \mathrm{Kg} \cdot \mathrm{m}^{-3}$} \\
\hline Dielectric Permittivity: & $\varepsilon_{11}^{\mathrm{S}}=12.75 \times 10^{-9} \mathrm{~F} / \mathrm{m}$ & $\varepsilon_{33}^{\mathrm{S}}=7.411 \times 10^{-9} \mathrm{~F} / \mathrm{m}$ \\
\hline Elastic compliances : & $\mathrm{s}^{\mathrm{E}}{ }_{11}=15.44 \times 10^{12} \mathrm{~m}^{2} / \mathrm{N}$ & $\mathrm{s}_{33}^{\mathrm{E}}=20.09 \times 10^{12} \mathrm{~m}^{2} / \mathrm{N}$ \\
\hline Charge coefficients: & $\mathrm{d}_{31}=-186 \mathrm{pC} / \mathrm{N}$ & $\mathrm{d}_{33}=425 \mathrm{pC} / \mathrm{N}$ \\
\hline
\end{tabular}

The resonance frequencies of the lower modes are given in Table 3.

Table-3 Frequencies of the three simulated modes of the beam [11]

\begin{tabular}{|l|l|}
\hline Modes & Frequency \\
\hline Mode 1 & $31.86 \mathrm{~Hz}$ \\
\hline Mode 2 & $171.52 \mathrm{~Hz}$ \\
\hline Mode 3 & $433.96 \mathrm{~Hz}$ \\
\hline
\end{tabular}

\section{MODEL-SSD TECHNIQUE}

Based on a modal model and SSD techniques of the controlled structure, the so-called Modal-SSDI has been developed [3][4]. This technique consists of synchronizing the switch sequence on a given modal coordinate instead of the voltage. It therefore combines the simplicity, the robustness and low power operation of SSDI techniques, with the possibility of mode targeting and precision of active control strategies.

As shown in Fig.3, Harari developed this semi-active method in the case of wide bandwidth excitations. This very efficient technique uses the modal description of structures and concentrates the modal energy on one or several target modes. A modal observer and a controller are used in the proposed method.

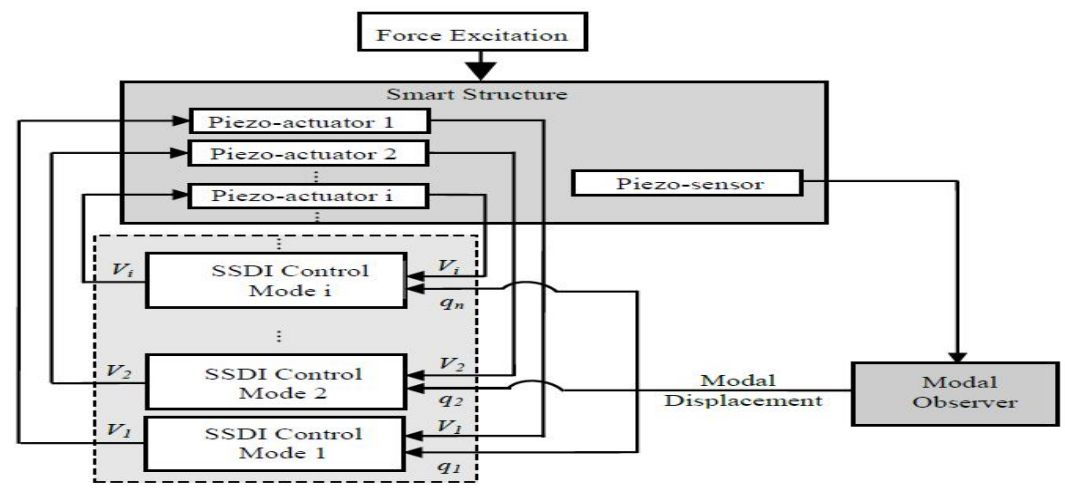

Figure 3. Schema of SSDI Modal [3][4] 


\section{MODEL SSDI-MAX METHOD}

Damping performance is strongly dependent on the piezoelectric voltage amplitude. The Modal-SSDI Max was developed [5][6][7], with the objective of maximizing the self-generated voltage amplitude, by a proper definition of the switch instants according to the chosen modal coordinate extremum. Compared with Modal-SSDI control method, the strategy of Modal-SSDI Max consists in delaying the switch instant to the next voltage extreme immediately, following the targeted modal coordinate extremum. It means that Modal-SSDI Max also relies on a modal model and modal observer permitting the calculation of the modal coordinates, to synchronize the switch with the targeted mode. In order to avoid a desynchronization with the target modal displacement, a time window is set up to limit the delay. Fig.4 illustrates the strategy of this technique in the various cases. The grey area materializes the time window, which is initiated for each extremum of the targeted modal coordinate. Point A, B, C and D illustrate the example of chosen switching time, which is indicated by the arrow.

- Point A: It is necessary to wait for the next positive maximum of the voltage to ensure the optimal voltage amplitude. Immediately switching would result in a wrong phase for the voltage of the piezoelectric actuator.

- Point B: Even if the voltage has the right sign, it is better to wait for the next voltage extremum to obtain a higher amplitude of the voltage.

- $\quad$ Point C: Voltage is decreasing at the beginning of the window, so it's better to switch immediately.

- Point D: If no voltage extremum with the correct sign is located in the time window, the switch should be closed at the end of the time window, thus limiting the resulting voltage phase shift.

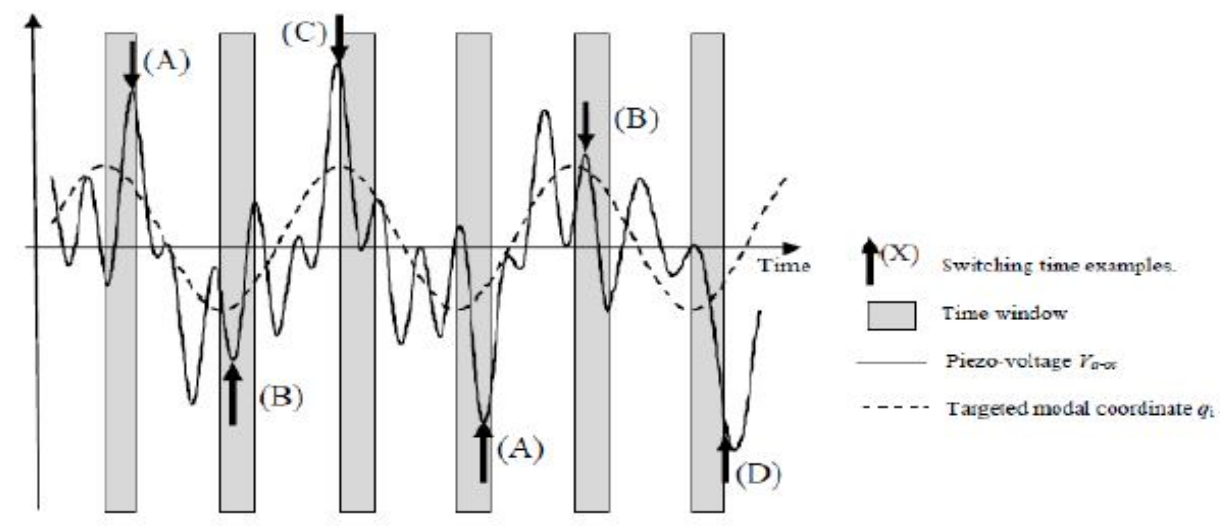

Figure 4. Strategy of Modal-SSDI Max vibration control [5]

\section{ENERGY TRANSFERRING TECHNOLOGY}

\section{A. SSDD (Synchronized Switch Damping With Diode)}

The energy harvesting techniques have experienced a great development over the last decades. As a result, a vibration damping system powered by harvested energy, using piezoelectric elements have been studied in the past few years. A global approach SSDD network, choosing the scavenge energy on low frequency modes (mode 1 and 2) are studied, to increase the damping effect of a high frequency mode (mode 3). All the piezoelectric elements are bonded on the smart beam and the structure is excited by a multi-sinusoidal source or by a pulse. This technique is a modal technique that requires a modal observer and a modal model of the structure. The various switches are closed on extremum of modal quantities ( $q 1$ to $q 3$ ) or on the sum of the modal quantities. Fig.5 present the circuit. 


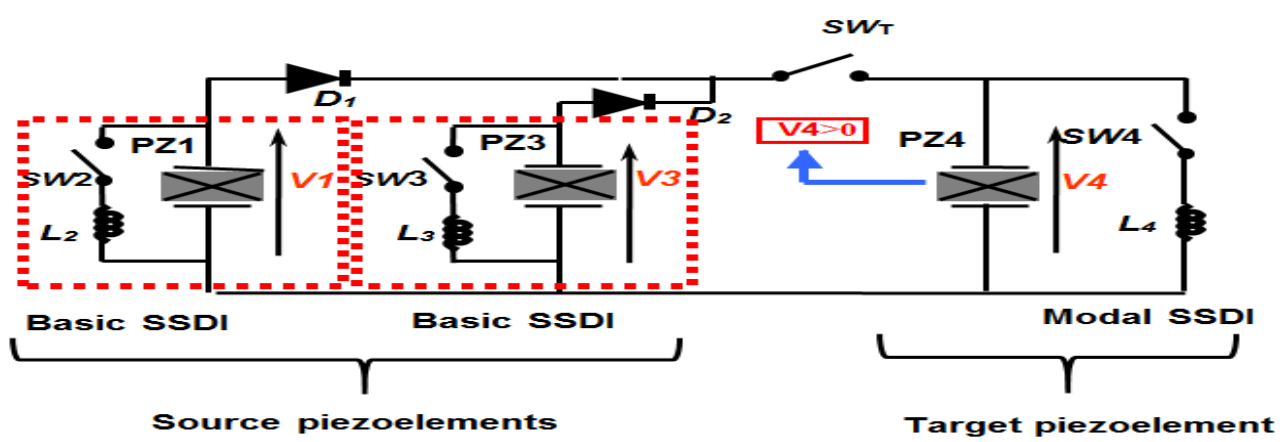

Figure5. Schematic of the SSDD network [8]

A more direct transfer would result in lower losses and better power flow. The use of a component switching naturally according to the source and target voltages, would simplify the overall transfer, making the choice of diodes natural. In this case, direct transfer takes place without any storage capacitances, and it can take the advantage of the unidirectional characteristic of the diode for the energy flow orientation. Moreover, it is interesting to note that many source elements $\left(\mathrm{PZ}_{1}\right.$ and $\left.\mathrm{PZ}_{3}\right)$ with the corresponding switching branches can be added in parallel in order to increase the total harvested energy flow.

A switching network is connected in parallel, in order to implement Modal-SSDI synchronized on the target mode. Whenever the voltages on the source piezoelectric elements are greater than the target one, the connecting diodes $D 1$ to $D 2$ can naturally switch-on in order to charge the target piezoelectric element. Note that if target piezoelectric voltage is negative, the diodes could switch-on and a current could flow in the diodes, resulting in the discharge of $V 4$ in the source piezoelectric elements. In order to prevent this unwanted case, an additional switch $S W T$ has been added. This switch is closed only when the target piezoelectric voltage is positive. Therefore, a power flow can be established only when the diode current is positive (natural behavior of the diodes) and when the target piezoelectric voltage is positive, thus resulting in a unidirectional power flow from the source piezoelectric elements. On the right side, nothing prevents charge flowing between the source piezoelectric elements. It is also remarkable that there are no discontinuities in the electric voltage changes, since due to the $(S W i, L i)$ networks, all the voltage changes are associated either with oscillating inversion processes or with the structure motion.

\section{B. SSDD-Max Technology}

The SSDD-Max technology has improved SSDD technique, their principles are the same, the difference between the two, is that in the method for SSDD technique, the switching network is connected in parallel in order to Implement Modal-synchronized SSDI on the target mode. By cons for SSDD-Max method, in the targeted mode switch method is SSDI-Max.

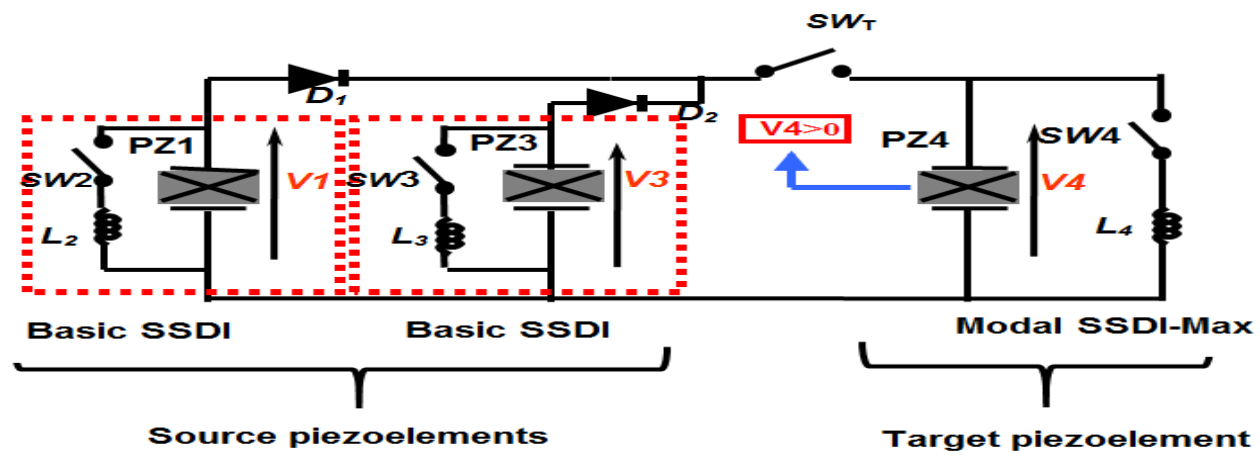

Figure 6. Schematic of the SSDD-Max network.

\section{SIMULATION}

The simulations are performed using the MATLAB/Simulink software environment. The step in SSDD-Max validation is the control of a single mode of the structure. This single mode control is considered with different types of multimodal excitations: multi-sinusoidal excitation or broadband excitation with a pulse. In all the cases, the target piezoelement PZ4 aims at the damping of the third mode and consequently, target switching is synchronized with the 
q3 modal coordinate. The other switches on the source side can be either triggered on specific modal coordinates, or on the voltages, or on a combination of modal coordinates in order to switch indifferently on all the source modes.

\section{Sinusoidal excitation}

In this case, the excitation is composed of the sum of three sine signals whose frequencies are those of the three modes of the structure. The interest of this type of excitation, relies in the fact that it is possible to adjust the energy associated with the various modal contents of the vibration.

The damping performance, using the optimized oriented SSDD-Max network is much more effective than ModalSSDI and SSDD network, which is shown in Fig.7. Hence, the damping performance of the Optimized Oriented SSDD-Max network is much more effective than other configurations. The only main drawbacks are the usual inversion losses still quantified by a $\gamma$ inversion coefficient equal to 0.7 , and the diodes voltage threshold (usually from 0.3 to $0.6 \mathrm{~V}$ ) which degrade the power flow performances for small voltage drops, between the source and target sides.

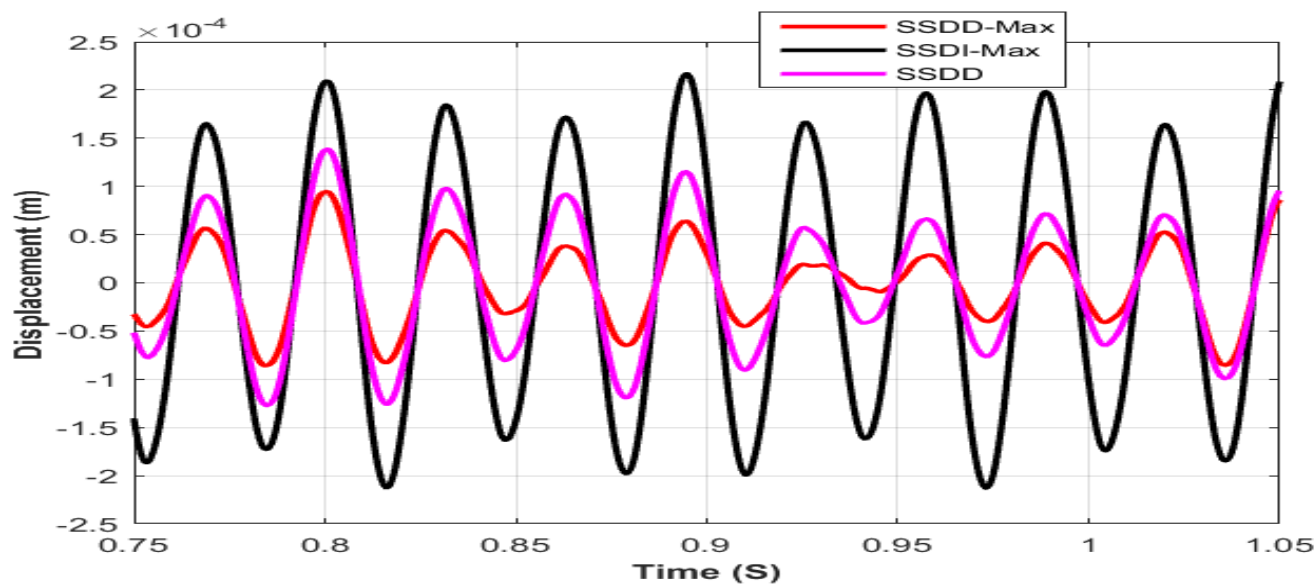

Figure 7. A comparison between the Modal SSDD-Max and the SSDD network damping performance on mode 3 in sinusoidal excitation.

\section{Pulse excitation}

The excitation is a wide frequency square force pulse, $50 \mu$ s long and corresponding to $5 \mathrm{~mJ}$ input energy.

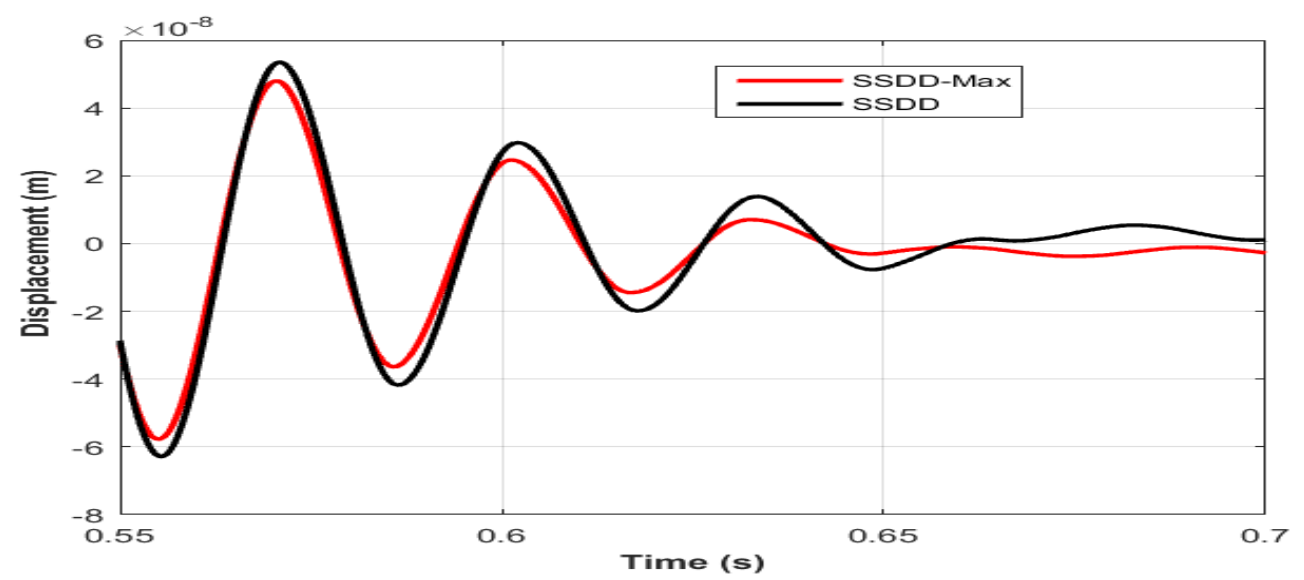

Figure 8. A comparison between the Modal SSDD-Max and the SSDD network damping performance on mode 3 in pulse excitation.

According to Fig.8, it is clearly illustrated that the use of the Optimized Oriented SSDD-Max network can induce much more damping effect, than the SSDD network configurations. 


\section{IIX.CONCLUSION}

In this paper, we propose the SSDD-Max technology, which is the improved SSDD network. This logic circuit uses multi-piezoelements with diodes to achieve the structural vibration control; by this logic circuit we increase the source modes energy transfer to the target mode by SSDI-Max technique. This circuit is more simplified and intelligent. Simulation results show that the dumping performances are better and much more effective, using the new logical circuit SSDD-Max network, than the Modal-SSDI and SSDD network for sinusoidal and impulse excitation.

\section{REFERENCES}

[1] Richard, C., Guyomar, D., Audigier, D., Ching, G., "Semi-passive damping using continuous switching of a piezoelectric device," Proceedings of SSM, Damping and Isolation, 3672, 104-111 (1999).

[2] Richard, C., Guyomar, D., Audigier, D., and Bassaler, H., "Enhanced Semi Passive Damping using Continuous Switching of a Piezoelectric Device on an Inductor," Proceedings SPIE SSM Conference, Passive Damping and Isolation, 3989, 288-299 (2000).

[3] Harari, S., Richard, C., Gaudiller, L., "Multimodal control of smart structures based on semi-passive techniques and modal observer," Motion and Vibration Control, 1, 113-122 (2009).

[4] Harari, S., Richard, C., Gaudiller, L., "Hybrid active/semi-active modal control of smart structures," Proceedings of SPIE SSM, 7288 (2009).

[5] Chérif, A., Richard, C., Guyomar, D., Belkhiat, S., Meddad, M., "Simulation of multimodal vibration damping of a plate structure using a modal SSDI-Max technique,” Journal of Intelligent Material Systems and Structures, 23(6), 675-689 (2012).

[6] Chérif, A., C. Richard, D. Guyomar, S. Belkhiat, M. Meddad, A. Eddiai, A. Hajjaji, "Modal SSDI-Max Technique of a Smart Beam Structure: Broadband Excitation”. Journal of Optoelectronics and Adva)nced Materials, Vol. 15, pp. 438 - 446 (2013).

[7] A. Chérif, C. Richard, D. Guyomar, S. Belkhiat, M. Meddad, A. Eddiai, A. Hajjaji, "Optimization of the Piezoelectric Transformer by the Non-Linear Methods". Optical and Quantum Electronics, DOI 10.1007/s11082-013-9712-2 (2013).

[8] D. Wu, D. Guyomar, C. Richard, "A new global approach using a network of piezoelectric elements and energy redistribution for enhanced vibration damping of smart structure”, Proceedings SPIE Conference, Active and Passive Smart Structures and Integrated Systems, Vol. 8688, 115 (2013).

[9] Badel, A. et al., "Piezoelectric vibration control by synchronized switching on adaptive voltage sources: towards wideband semi-active damping," Acoustical Society of America, 119, 2815-2825 (2006).

[10] Lefeuvre, E., Badel, A., Petit, L., Richard, C., Guyomar, D., "Semi-passive Piezoelectric Structural Damping by Synchronized Switching on Voltage Sources," Journal of Intelligent Material Systems and Structures, 17, 653-660 (2006).

[11] A. Chérif, C. Richard, D. Guyomar, S. Belkhiat, M. Meddad, A. Eddiai, A. Hajjaji, "Modal SSDI-Max Technique of a Smart Beam Structure: Broadband Excitation”. Journal of Optoelectronics and Advanced Materials, Vol. 15, pp. 438 - 446 (2013). 